Valoración social del docente universitario: una perspectiva fenoménica en IEU del estado Falcón

Luís Piña, Lesdybeth Rodríguez y Blanquita García ( pp. 102-121)

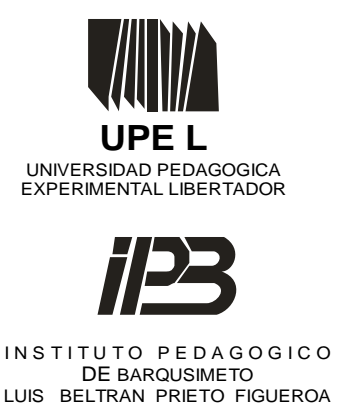

DE BARQUSIMETO
LUIS BELTRAN PRIETO FIGUEROA

BARQUISIMETO - EDO. LARA - VENEZUELA

NUEVA ETAPA

FORMATO ELECTRÓNICO

DEPOSITO LEGAL: ppi201002LA3674

ISSN: 2244-7296

Volumen 18 № 3

Septiembre-Diciembre 2014

VALORACIÓN SOCIAL DEL DOCENTE UNIVERSITARIO: UNA PERSPECTIVA FENOMÉNICA EN IEU DEL ESTADO FALCON

PROFESSORS' SOCIAL ROLE VALORIZATION IN FALCON STATE:

A PHENOMENOLOGICAL PERSPECTIVE

Órgano Divulgativo de la Subdirección de Investigación y Postgrado del Instituto Pedagógico de Barquisimeto "Luis Beltrán Prieto 


\section{VALORACIÓN SOCIAL DEL DOCENTE UNIVERSITARIO: UNA PERSPECTIVA FENOMÉNICA EN IEU DEL ESTADO FALCÓN}

\section{PROFESSORS' SOCIAL ROLE VALORIZATION IN FALCON STATE: A PHENOMENOLOGICAL PERSPECTIVE}

$\underline{E N S A Y O}$

Recibido: 02-10-2014
Luis Eduardo Piña López

UPT de Falcón "Alonso Gamero" Lesdybeth Rodríguez ** Blanquita C. García G.****

Universidad del Zulia

Aceptado: 01-12-2014

\section{RESUMEN}

El presente ensayo pretende interpretar la valoración social de los docentes universitarios en dos Instituciones de Educación Universitaria (IEU) del estado Falcón. Partió de un intercambio subjetivo, producto de una actividad de formación continua en el contexto profesional y de la autopercepción. Se aplicaron entrevistas en profundidad con su posterior categorización para el análisis y contraste, agrupadas por áreas tipológicas; luego como macro-categorías saturadas. Entre los hallazgos fue posible develar los logros de trayectoria académica y posición docente, aunado a la actuación centrada en el profesionalismo que contrasta con los requerimientos socio económicos, la calidad de vida y el compendio de saberes generador del perfil $y$ emprendimiento educativo, al tiempo que los informantes destacaron como requerimiento, la necesidad de valorización y formación permanente para la constante transformación personal-social.

Descriptores: valoración social, fenomenología, docencia universitaria, experiencia profesional.

\begin{abstract}
This essay aims to interpret professors' social role valorization from two universities in Falcón State. This essay was conceived during a professional development activity based on selfperception in the professional field. In-depth interviews organized first according to typology and later as saturated macro-structures were applied. Data were categorized, contrasted and analyzed. Results highlight academic achievement and teacher's ranks in an environment where life quality and a squeezing economy have put professionalism and knowledge (the ignition to educational venture) to fire and sword. Informants insisted on the need to be valued under these circumstances and to keep constantly training for a personal and social transformation.
\end{abstract}

\footnotetext{
*Doctorante en Planificación y Gestión del Desarrollo Regional (LUZ, Núcleo Punto Fijo). MSc. en Gerencia de Calidad y Productividad. Ingeniero Mecánico. Profesor Agregado de la UPT de Falcón Alonso Gamero. E-mail: luispinalopez@gmail.com ** Doctorante en Planificación y Gestión del Desarrollo Regional (LUZ, Núcleo Punto Fijo). MSc. en Corrosión. Ingeniero Químico. Profesora Asociada de la Universidad del Zulia (LUZ, Núcleo Punto Fijo). Núcleo Punto Fijo. E-mail: lesdybeth@gmail.com

*** Doctora en Ciencias, mención: Investigación. MSc. en Planificación y Gerencia de Ciencia y Tecnología. Licenciada en Educación. Profesora Titular a Dedicación Exclusiva en la Universidad del Zulia (LUZ, Núcleo Punto Fijo). Investigadora acreditada PPI/PEII C (ONCTI). Editora Jefe de la Revista Multiciencias. E-mail: blangargar@gmail.com
} 


\section{Valoración social del docente universitario: una perspectiva fenoménica en IEU del estado Falcón \\ Luís Piña, Lesdybeth Rodríguez y Blanquita García ( pp. 102-121)}

\section{INTRODUCCIÓN}

Hablar de las vivencias que los docentes universitarios han acumulado en la región falconiana en los últimos años, implica interactuar con ellos para conocer sus puntos de vista acerca de sus experiencias, desde sus inicios laborales en las Instituciones de Educación Universitaria (IEU) que ahora representan y, así, intentar comprender sus planteamientos más resaltantes. Es un acercamiento en lo introspectivo que puede abordarse cualitativamente.

De acuerdo con Martínez (2004), la metodología cualitativa a diferencia de la cuantitativa no parte de un problema específico, sino que aborda un área problemática poco definida en la que pueden existir un sinnúmero de problemas entrelazados que sólo será posible identificarlos una vez esté iniciada y avanzada la investigación. Esto es, la problemática planteada se presenta ante el investigador como un horizonte de problemas y es tarea de éste definir claramente la intencionalidad de su investigación, aquello que quiere o lo que no quiere investigar.

Por su parte, Yuni y Urbano (2005), refieren que el formularse preguntas permite acercarse con la mayor concreción posible al fenómeno a investigar, para revelar aquello que le da significado y sentido desde la complejidad de la realidad humana. Así también, Piñero y Rivera (2013), en continua vigilancia de la coherencia paradigmática, sugieren seleccionar la metodología de aproximación al problema que se considere más adecuada según los fines y la realidad a investigar.

Al partir de estas premisas, se presenta este trabajo cuya intencionalidad está dirigida a recrear las particularidades de los profesores universitarios, desde su recogida de datos hasta el posterior análisis cualitativo, aplicando la técnica de la entrevista en profundidad. Esta técnica, según Taylor y Bogan (1992), requiere que el investigador establezca contacto directo con las personas que participan en calidad de informantes. Así la información a ser revelada, consiste principalmente en conceptos, percepciones, imágenes mentales, creencias, emociones, interpretaciones, experiencias y vivencias, en el marco de un contexto social específico, las cuales son accesibles al investigador a través del diálogo y

Revista EDUCARE, Volumen 18, Número 3, Septiembre - Diciembre 2014. ISSN: 2244-7296 Página 104 


\section{Valoración social del docente universitario: una perspectiva fenoménica en IEU del estado Falcón \\ Luís Piña, Lesdybeth Rodríguez y Blanquita García ( pp. 102-121)}

manifestadas en el lenguaje del sujeto informante. Para estos fines, se estableció como temática de abordaje la valoración social del docente universitario, por considerarse como una situación vivenciada y experimentada, facilitando la interacción requerida en cuanto a la ubicación de un contexto común para los sujetos informantes y los investigadores.

En cuanto a la organización de este artículo, se intenta agruparlo en cuatro áreas: en la primera se realiza una aproximación inicial al objeto de estudio donde se presenta el propósito inicial, el cual parte de la percepción del docente universitario sobre la valoración que le da la sociedad a su profesión, así como la caracterización o contextualización del objeto de estudio. En la segunda, se describen teorías, características de los informantes, y lógica metódica. Posteriormente, se detalla el proceso de obtención de la información como sistematización del mismo a través de su codificación-categorización. Finalmente, se construyen los significados que emergieron de los sujetos, obtenidos a partir de la compresión de su discurso, es decir, de las experiencias vividas como docentes.

Para estos fines, se estableció como temática de abordaje la valoración social del docente universitario, por considerarse como una situación vivenciada y experimentada que facilita la interacción requerida en cuanto a la ubicación del contexto común tanto para los sujetos informantes así como los investigadores.

Conviene entonces, realizar la contextualización del objeto de estudio de la presente investigación centrada en los docentes universitarios, específicamente en la apreciación vivencial de su quehacer profesoral en IEU del estado Falcón, sin perder de vista las diferentes variables que intervienen en el proceso. La ruta epistemológica versó en la investigación cualitativa con método fenomenológico, al contrastar la realidad presentada por tres docentes, siendo la realidad temática: la percepción y las vivencias de los docentes su condición de profesores universitarios, con ubicación geográfica en la entidad falconiana.

En este orden de ideas destaca que esta valoración social de las experiencias y vivencias tienen varios factores de referencia o puntos de encuentro que señalan a la situación laboral como indicador de las diferencias. Se puede afirmar entonces que la referida situación laboral de los docentes universitarios en los últimos años en 


\section{Valoración social del docente universitario: una perspectiva fenoménica en IEU del estado Falcón \\ Luís Piña, Lesdybeth Rodríguez y Blanquita García ( pp. 102-121)}

Latinoamérica y de forma especial en Venezuela, ha estado marcada por una multiplicidad de elementos, entre los cuales destaca, la situación económica, política, social, cultural y de valores por la cual atraviesa el país y como muestra tangible basta leer la prensa o ver los noticieros, tanto nacionales como internacionales que indican una situación que continuamente se vuelve más incierta y más compleja. Y es en este escenario donde los docentes interactúan y van marcando la pauta y condicionando su forma de pensar, sentir y actuar.

\section{DESCRIPCIÓN DE LA TEMÁTICA}

Realizando una breve revisión documental sobre el tema, Guevara y Domínguez (2011), resaltan como consideración recurrente la calidad profesional del docente universitario como pieza clave y determinante de la particularidad del sistema educativo en lo social; a lo que Marchesi y Díaz (2008), señalan que está tradicionalmente asociada a valores como la responsabilidad, compromiso, dedicación, respeto, igualdad, honestidad, tolerancia, paciencia, justicia y prudencia, entre otros.

También destaca el trabajo de Ávalos y otros (2010), relacionado con la profesión docente, el ejercicio de la misma y la forma como los docentes enfrentan los cambios y reformas en los sistemas educacionales. En este sentido, el radio de acción gira en torno al docente y su estatus profesional. No obstante, queda claramente establecido que el carácter y la relevancia profesional de la docencia, en modo alguno, es tema de discusión. Ahora bien, lo que si fue objeto de revisión fue el modo como se percibe a los docentes y se valora socialmente su trabajo, lo cual indiscutiblemente incide en la forma en cómo los docentes se ven, se perciben y se interpretan a sí mismos, lo cual afecta en la ejecución de su trabajo docente.

En este orden de ideas, destaca la profesión docente como muy importante, profesionales que valoran su trabajo, pero se sienten "acorralados por las demandas y presiones externas y con un reconocimiento social ambiguo". (Avalos y otros, ob.cit.; p. 260). Esto sin olvidar las evidencias tangibles que se traducen en el ambiente y condiciones 


\section{Valoración social del docente universitario: una perspectiva fenoménica en IEU del estado Falcón \\ Luís Piña, Lesdybeth Rodríguez y Blanquita García ( pp. 102-121)}

de trabajo, las reformas constantes, la pérdida paulatina, no declarada, pero si evidenciada de la autonomía docente, los contenidos curriculares y las evaluaciones estandarizadas y como último pero muy importante, porque se traduce en la calidad y condiciones de vida de los docentes, los salarios insuficientes y alejados de la realidad social y del gran compromiso que el docente tiene.

En la revisión realizada por los autores antes referidos, quedó como evidencia tangible la gran importancia del reconocimiento de los referentes sociales de su entorno, de allí que la opinión y valoración pública resulta importante para la autovaloración. Coinciden en que la apreciación de las condiciones en las cuales se desempeñan los docentes universitarios no es la misma, ha cambiado significativamente, y los cambios se han visto marcados por muchos factores, entre los que destacan la globalización que lideran las variaciones originadas por la sociedad de la información, además de cambios económicos, políticos y sociales.

En cuanto a los aspectos clave, como son la satisfacción o insatisfacción en la profesión, destacan las condiciones físicas, el desarrollo personal, el aspecto económico y su incidencia en las vivencias de los profesores. Al respecto, se tiene el trabajo de Rodríguez y otros (2010) quienes desde la Universidad de Oriente reportan un contraste en un quinquenio (2005-2009), obteniendo de la consulta a 200 docentes universitarios en muy bajo nivel de satisfacción con evidente significancia estadística en el condicionante económico, además del gran decaimiento en el desarrollo personal de los docentes.

En otras investigaciones, como el documento final de la Organización de Estados Iberoamericanos - OEI (2010), se pone en evidencia que si bien su profesión le permite satisfacciones existe un alto grado de insatisfacción en estos, principalmente a causa de su percepción de no contar con un reconocimiento social deseable atendiendo las condiciones económicas ajustadas, la falta de oportunidades, y escasas ayudas para su formación inicial o continua. A la luz de esta descripción, resulta interesante la proposición de una pregunta generadora como es la de referirse a la percepción de la profesión docente vista por la sociedad hoy día. Surge entonces la inquietud de escuchar desde la voz de los actores, sus experiencias que a su vez permitan delinear las perspectivas desde una visión de conjunto.

Revista EDUCARE, Volumen 18, Número 3, Septiembre - Diciembre 2014. ISSN: 2244-7296 Página 107 


\section{Valoración social del docente universitario: una perspectiva fenoménica en IEU del estado Falcón \\ Luís Piña, Lesdybeth Rodríguez y Blanquita García ( pp. 102-121)}

De esta forma, se ubica la investigación en un contexto que resulta familiar tanto para los investigadores como para los sujetos informantes, permitiendo iniciar la interacción sobre la base de vivencias comunes, siguiendo la lógica metódica cualitativa con características reflexivo-recursivas para el intercambio dialógico entre actores y la comunicación de subjetividades.

En este sentido, una forma esquemática de fases parte del establecimiento de la valoración docente (fase reflexiva), la planificación requerida para la realización de las entrevistas (fase de planificación), así como el proceso de recogida y análisis de los datos cualitativos (fase de recolección de la información), lo cual da lugar a la construcción de las interpretaciones (fase de elaboración del informe final), haciendo obvio lo que inicialmente se aprecia como "invisible". Inclusive, las primeras tres fases pueden corresponder a una primera etapa, y la cuarta fase a una etapa final. Reflexivamente, se hace imprescindible declarar nuevamente la intencionalidad indagatoria, que es la de interpretar las situaciones que acontecen en el entorno de los docentes universitarios, sus experiencias en el tiempo que han dedicado a sus funciones educativas y de investigación desde que forman parte de sus instituciones, el contexto donde se desenvuelven y las percepciones con que se encuentran.

Como aval de referencia a lo expresado en el párrafo anterior, se encuentran los trabajos de Córdova (2011) quien aborda el tema de las motivaciones que llevan a un individuo a seleccionar la carrera docente, aunado a los factores que influencian la motivación en su quehacer cotidiano. Lo preocupante viene luego, cuando la carrera escogida involucra tanto pero se mantiene estática en cuanto a la valoración del encargado de la formación.

Por su parte, García y otros (2013) mencionan como acertado que ante la problemática existente en el país a la cual no escapa la educación y de forma especial la formación de formadores; los que se preparan para el ejercicio de la profesión docente considerando como relevante el establecimiento de criterios, normas o directrices homogéneas, permitan la unificación de criterios en la formación y en los resultados tangibles en la sociedad. En este sentido, el código ético del profesional de la docencia 


\section{Valoración social del docente universitario: una perspectiva fenoménica en IEU del estado Falcón \\ Luís Piña, Lesdybeth Rodríguez y Blanquita García ( pp. 102-121)}

"debe estar estrechamente vinculado con las realidades sociales, donde se expongan las competencias, el perfil y el compromiso que deben asumir los docentes frente a los educandos, familia, escuela, comunidad, sociedad y país.” (p.62).

\section{DESCRIPCIÓN METODOLÓGICA}

El trabajo del cual se desagrega el presente artículo se desarrolla tomando en consideración el enfoque cualitativo interpretativo con análisis de contraste que parte de la apreciación de las vivencias de tres docentes universitarios del estado Falcón, utilizando para ello el método fenomenológico, el cual parte de la realidad conocida y permite la concatenación de ideas para la estructuración de un análisis descriptivo basado en las experiencias intersubjetivas. En este sentido, se pretende un análisis aproximado a los significados que dan éstos docentes a sus vivencias transformadas en experiencias, así como los elementos incentivadores para el ejercicio de la profesión docente y la huella que han dejado o influenciado en el ejercicio como docentes universitarios.

Dada la naturaleza epistémica de la investigación cualitativa, ésta requiere de la participación de sujetos que permitan al investigador el acceso a información relevante sobre la temática, por lo que quienes participaron como informantes fueron escogidos por su relación directa con el ámbito abordado, considerando los siguientes criterios: docentes universitarios con reconocida experiencia (mayor a 10 años), dedicación a tiempo completo o exclusiva, e interés demostrado en el área objeto de esta investigación.

En este sentido, la lógica-metódica fenomenológica seguida fue de orientación husserliana, donde primeramente se examinaron los contenidos de la conciencia para luego determinar sus tipologías reales e ideales. Posteriormente, suspender la conciencia fenomenológica para posibilitar la descripción de purezas relativas tal cual como son los fenómenos (Husserl, 1962).

De esta forma, se seleccionaron tres sujetos informantes identificados con las siglas: E-1, E-2, y E-3; representando a profesores ordinarios de IEU de la región falconiana, con más de doce (12) años de ejercicio en educación superior, la obtención de varios grados de

Revista EDUCARE, Volumen 18, Número 3, Septiembre - Diciembre 2014. ISSN: 2244-7296 Página 109 


\section{Valoración social del docente universitario: una perspectiva fenoménica en IEU del estado Falcón \\ Luís Piña, Lesdybeth Rodríguez y Blanquita García ( pp. 102-121)}

instrucción entre pregrado y posgrado, así como acreditada experiencia en la administración de unidades curriculares. Es importante mencionar, que los informantes participaron de forma voluntaria manifestando su total disposición a ser entrevistados bajo el consentimiento de emplear la información con fines enteramente científicos, perteneciendo estos a la Universidad del Zulia (Punto Fijo), y la Universidad Politécnica Territorial de Falcón Alonso Gamero, situada en Coro.

La recolección de datos se logró a través de la realización de entrevistas en profundidad a los sujetos informantes elegidos para tal fin. Al respecto, Corbetta (2007) señala que esta técnica cualitativa pretende conocer la individualidad de la persona entrevistada, comprender sus categorías mentales, interpretaciones, percepciones y sentimientos, así como los motivos de sus actos. Previamente, autores como Taylor y Bogan (Ob.cit.), expresan que se trata de "reiterados encuentros cara a cara entre el investigador y los informantes, encuentros estos dirigidos a la comprensión de las perspectivas que tienen respecto a sus vidas, experiencias o situaciones, tal y como las expresan con sus propias palabras" (p. 100). Según Álvarez-Gayou (2005), se puede considerar a la entrevista como una conversación que tiene una estructura y un propósito.

A los fines de esta realización, se decidió aplicar la entrevista de forma individual y semiestructurada debido a la complejidad de las dimensiones que comprenden la valoración social de la profesión docente como problema, lo que pudiera generar una cantidad considerable de categorías que dificultarían el análisis de los resultados. Es así, como se concibió un guión de entrevista que transitó resumidamente en lo siguiente: a) Inicios como docente universitario, b) Experiencias vividas al ingresar como docente universitario, c) Significado del rol docente, d) Planes de formación y actualización, e) Escalafón universitario, f) Percepciones de la comunidad estudiantil frente a usted, g) La profesión docente vista hoy día, h) Valoración del profesor universitario, i) Calidad de vida del docente universitario, j) ¿Qué es para Usted ser docente universitario?.

Por tanto, para la realización de la entrevista se siguió atentamente el siguiente esquema general protocolar a los fines de sostener un clima cordial y afable con los sujetos versionantes: 


\section{Valoración social del docente universitario: una perspectiva fenoménica en IEU del estado Falcón \\ Luís Piña, Lesdybeth Rodríguez y Blanquita García ( pp. 102-121)}

1. Presentación: De forma previa a la realización de la entrevista se realizó la descripción de la temática en torno a la cual estaría centrada la misma, así como la intencionalidad de la investigación. Esta preparación inicial, permitió al entrevistado aclarar cualquier duda tanto sobre la temática de la entrevista así como sobre el procedimiento para su realización.

2. Interacción: El guión elaborado indicó los temas a ser abordados en la entrevista, sin embargo éste no determinó la secuencia de las preguntas. El investigador permitió al entrevistado la amplitud en sus respuestas, realizando preguntas cortas para guiar la conversación, respetando en todo momento su ritmo de respuesta, su velocidad de pensamiento y su expresión verbal.

3. Cierre: El investigador presentó un pequeño resumen de los temas tratados y, cuando fue necesario, se realizó una revisión y profundización de algunos temas puntuales de acuerdo con el resultado de la entrevista. Se agradeció la participación voluntaria del informante, acordándose un próximo encuentro de ser necesario.

4. Palabras finales: Se le indicó a los informantes que una vez transcrita la entrevista tendrán la oportunidad de leerla y realizar comentarios adicionales, en caso de considerarlo necesario, así como el agradecer su participación.

De esta manera, las entrevistas fueron grabadas en formato de audio digital, para luego ser transcritas en su totalidad, sin resumir ni alterar las expresiones utilizadas por el informante. Durante la realización de la entrevista se tomaron notas para facilitar la formulación de nuevas preguntas y seguir la secuencia de las mismas, así como de la reacción del entrevistado hacia ciertas temáticas ayudando a dar sentido a la información recabada.

\section{Presentación de hallazgos}

De acuerdo con Yuni y Urbano (Oob.cit.), "el análisis de datos es reducir, categorizar, clarificar, sintetizar y comparar la información con el fin de obtener una información lo más completa posible del fenómeno observado” (p. 253). Dicho análisis se 


\section{Valoración social del docente universitario: una perspectiva fenoménica en IEU del estado Falcón \\ Luís Piña, Lesdybeth Rodríguez y Blanquita García ( pp. 102-121)}

entrelazó con la lógica-metódica descrita para conservar su coherencia con la significancia fenomenológica.

Para los mencionados autores, el análisis de datos consiste en encontrar tramas de sentido en los textos generados a partir del material discursivo recolectado. En el proceso de codificación abierta, resaltada por Strauss y Corbin (2002) como una fase crítica del proceso investigativo cualitativo, el investigador descompone los datos en partes más discretas para luego proceder a un examen exhaustivo en la búsqueda de similitudes. Con base en las semejanzas encontradas, agruparlos en conceptos más abstractos denominados categorías, que a su vez definen como una representación abstracta de un acontecimiento, objeto o acción/interacción, identificado como significativo en los datos de acuerdo con el contexto abordado, siendo esto lo más importante al momento de desarrollar sus propiedades o dimensiones.

Luego de recolectada, transcrita, revisada y organizada la información, se procedió a su análisis a través de la construcción de categorías mediante proceso de codificación abierta. A saber, las entrevistas fueron transcritas en un formato doble columna donde los dos tercios derechos de la página fueron utilizados para la transcripción como tal, con numeración de líneas del texto; el tercio restante fue destinado a la categorización (etiquetas codificadas de sentido especial-práctico).

Se procedió entonces a dividir los contenidos en porciones o unidades temáticas. Esto se realizó empleando la clasificación por áreas tipológicas (o temáticas), de acuerdo con Goetz y LeCompte (1988), quienes señalan lo siguiente:

El análisis tipológico consiste en dividir todo lo que se observa en grupos o categorías sobre la base de alguna regla de descomposición de los fenómenos. Las tipologías se pueden diseñar a partir de un marco teórico o conjunto de proposiciones o bien de forma más mundana, desde las concepciones cotidianas o del sentido común (p. 189).

Cabe resaltar, que la clasificación por áreas tipológicas fue tratada inicialmente por Patton (2001), refiriéndose a un ordenamiento en áreas del conocimiento de estrecha relación con el guión de entrevistas; esto para facilidad del proceso de categorización. Para esto, en el formato de transcripción de las entrevistas se realizó el rotulado de frases y

Revista EDUCARE, Volumen 18, Número 3, Septiembre - Diciembre 2014. ISSN: 2244-7296 Página 112 


\section{Valoración social del docente universitario: una perspectiva fenoménica en IEU del estado Falcón \\ Luís Piña, Lesdybeth Rodríguez y Blanquita García ( pp. 102-121)}

oraciones utilizando colores diferentes (acromatizado) de acuerdo con las áreas tipológicas y categorías emergentes del proceso cualitativo. Es importante mencionar que, dada la naturaleza práctica para la selección y agrupación de las frases en las diferentes tipologías y categorías, el proceso fue efectuado siguiendo el sentido común soportado por la experiencia de los investigadores (Schutz, 1974).

A efecto de síntesis organizativa, tales tipologías y categorías descubiertas en las entrevistas se muestran en los cuadros 1, 2, y 3; los cuales se presentan a continuación, resaltando que los códigos entre paréntesis seguidos luego de cada categoría inician con el área tipológica (A, B, C, D, E, F), añadiéndoseles iníciales identificativas. Más adelante, en la nomenclatura utilizada los dos primeros lugares corresponden al código de la entrevista analizada, lo que permite identificar cada sujeto informante (E1, E2, y E3). Los siguientes lugares, indican la categoría correspondiente de acuerdo con el código empleado.

\section{Cuadro 1}

\section{Áreas tipológicas y categorías encontradas en la Entrevista $\mathbf{N}^{\circ} 1$}

\begin{tabular}{|c|c|c|}
\hline $\begin{array}{l}\text { (A) Experiencia } \\
\text { (Amarillo) }\end{array}$ & $\begin{array}{l}\text { (B) Valores } \\
\text { (Turquesa) }\end{array}$ & $\begin{array}{l}\text { (C) Sensorial } \\
\text { (Verde lima) }\end{array}$ \\
\hline $\begin{array}{l}\text { Trayectoria académica (ATA) } \\
\text { Experiencia profesional (AEP) } \\
\text { Experiencia docente (AED) } \\
\text { Amplitud profesional (AAP) }\end{array}$ & $\begin{array}{l}\text { Agradecimientos (BAG) } \\
\text { Actitud centrada (BAC) } \\
\text { Ética profesional (BEP) } \\
\text { Interés profesional (BIP) }\end{array}$ & $\begin{array}{l}\text { Percepción descentrada (CPD) } \\
\text { Desincorporación (CDI) } \\
\text { Percepción de la calidad (CPC) } \\
\text { Percepción falso-educativa (CPF) } \\
\text { Internalizar (CIN) } \\
\text { Necesidades del docente (CND) }\end{array}$ \\
\hline $\begin{array}{l}\text { (D) Conocimientos } \\
\text { (Rojo) }\end{array}$ & $\begin{array}{c}\text { (E) Organización } \\
\text { (Azul) }\end{array}$ & $\begin{array}{l}\text { (F) Antecedentes } \\
\text { (Oliva) }\end{array}$ \\
\hline $\begin{array}{l}\text { Reconocimiento (DRE) } \\
\text { Escalafón (DEN) } \\
\text { Saberes (DSA) } \\
\text { Explicación contextual (DEC) }\end{array}$ & $\begin{array}{l}\text { Contexto (ECO) } \\
\text { Concurso (ECC) } \\
\text { Planes de formación (EPF) } \\
\text { Ritmo laboral (ERL) } \\
\text { Evaluación del personal (EEV) }\end{array}$ & $\begin{array}{l}\text { Historia (FHI) } \\
\text { Actitudes educo-descentradas (FAD) } \\
\text { Homologación (FHO) }\end{array}$ \\
\hline
\end{tabular}

Fuente: Elaboración de los autores a partir de las entrevistas (2015)

Por esto, se reconfiguran los códigos (como por ejemplo: E1ATA:8-9). Luego de los dos puntos, se indica el número o los números de línea que permite ubicar la frase en el formato utilizado para la transcripción de la entrevista. Es decir, la nomenclatura refleja que los dos primeros caracteres corresponden al código de la entrevista (E1), los tres siguientes 
al código de la categoría (ATA), dos puntos separadores, y finalmente el número (o rango) de línea(s), (8-9). Como aspecto estético, se colocó de forma acromatizada tales códigos al final de cada frase de significación en cuadros reorganizados.

\section{Cuadro 2}

\section{Áreas tipológicas y categorías encontradas en la Entrevista $\mathbf{N}^{\circ} 2$}

\begin{tabular}{|c|c|c|}
\hline $\begin{array}{l}\text { (A) Experiencia } \\
\text { (Amarilld) }\end{array}$ & $\begin{array}{l}\text { (B) Valores } \\
\text { (Turquesa) }\end{array}$ & $\begin{array}{l}\text { (C) Sensorial } \\
\text { (Verde lima) }\end{array}$ \\
\hline $\begin{array}{l}\text { Experiencia docente (AED) } \\
\text { Amplitud profesional (AAP) }\end{array}$ & $\begin{array}{l}\text { Agradecimientos (BAG) } \\
\text { Actitud centrada (BAC) } \\
\text { Ética profesional (BEP) } \\
\text { Interés profesional (BIP) } \\
\text { Vocación (BVO) } \\
\text { Descrédito (BDE) }\end{array}$ & $\begin{array}{l}\text { Percepción descentrada (CPD) } \\
\text { Percepción de la calidad (CPC) } \\
\text { Percepción falso-educativa (CPF) } \\
\text { Internalizar (CIN) } \\
\text { Necesidades del docente (CND) } \\
\text { Incomodidad (CID) } \\
\text { Satisfacción (CSA) } \\
\text { Indecisión (CIO) } \\
\text { Negligencia (CNE) }\end{array}$ \\
\hline $\begin{array}{l}\text { (D) Conocimientos } \\
\text { (Rojo) }\end{array}$ & $\begin{array}{c}\text { (E) Organización } \\
\text { (Azul) }\end{array}$ & $\begin{array}{c}\text { (F) Antecedentes } \\
\text { (Oliva) }\end{array}$ \\
\hline $\begin{array}{l}\text { Reconocimiento (DRE) } \\
\text { Saberes (DSA) } \\
\text { Explicación contextual (DEC) } \\
\text { Deficiencia de formación (DDF) }\end{array}$ & $\begin{array}{l}\text { Contexto (ECO) } \\
\text { Concurso (ECC) } \\
\text { Evaluación del personal (EEV) }\end{array}$ & $\begin{array}{l}\text { Historia (FHI) } \\
\text { Actitudes educo-descentradas (FAD) } \\
\text { Oportunidad (FOP) }\end{array}$ \\
\hline
\end{tabular}

Fuente: Elaboración de los autores a partir de las entrevistas (2015)

Así se logra completar un proceso de codificación tipológica categorizada para la primera etapa (cuadro 4), que a su vez servirá de referente para la etapa final, la cual es abducida, con categorías emergentes a partir de las encontradas como saturadas, que esta vez representan las denominadas macro-categorías.

\section{Cuadro 3}

\section{Áreas tipológicas y categorías encontradas en la Entrevista $\mathbf{N}^{\circ} 3$}

\begin{tabular}{|c|c|c|}
\hline $\begin{array}{l}\text { (A) Experiencia } \\
\text { (Amarillo) }\end{array}$ & $\begin{array}{l}\text { (B) Valores } \\
\text { (Turquesa) }\end{array}$ & $\begin{array}{l}\text { (C) Sensorial } \\
\text { (Verde lima) }\end{array}$ \\
\hline $\begin{array}{l}\text { Trayectoria académica (ATA) } \\
\text { Amplitud profesional (AAP) } \\
\text { Apoyo profesional (AAY) }\end{array}$ & $\begin{array}{l}\text { Agradecimientos (BAG) } \\
\text { Actitud centrada (BAC) } \\
\text { Ética profesional (BEP) } \\
\text { Descrédito (BDE) } \\
\text { Acercamiento (BAO) }\end{array}$ & $\begin{array}{l}\text { Percepción de la calidad (CPC) } \\
\text { Necesidades del docente (CND) }\end{array}$ \\
\hline $\begin{array}{l}\text { (D) Conocimientos } \\
\text { (Rojo) }\end{array}$ & (E) Organización & $\begin{array}{c}\text { (F) Antecedentes } \\
\text { (Oliva) }\end{array}$ \\
\hline $\begin{array}{l}\text { Escalafón (DEN) } \\
\text { Explicación contextual (DEC) } \\
\text { Deficiencia de formación (DDF) } \\
\text { Imprecisión (DIM) }\end{array}$ & $\begin{array}{l}\text { Contexto (ECO) } \\
\text { Planes de formación (EPF) }\end{array}$ & Historia (FHI) \\
\hline
\end{tabular}

Fuente: Elaboración de los autores a partir de las entrevistas (2015) 


\section{Valoración social del docente universitario: una perspectiva fenoménica en IEU del estado Falcón \\ Luís Piña, Lesdybeth Rodríguez y Blanquita García ( pp. 102-121)}

A decir de la etapa final, la misma se comprende de un proceso abducido como producto de la revisión de las categorías encontradas con mayor saturación o fenómeno de repitencia en las entrevistas; y que nuevamente a partir de las voces de los versionantes se descomponen frases o términos agrupantes temáticamente.

\section{Cuadro 4}

Descripción de frases de significación por categorías develadas de mayor saturación

\begin{tabular}{|c|c|c|}
\hline Áreas tipológicas & Cate & Descripción detallada por frases de significación \\
\hline \multirow{3}{*}{$\begin{array}{l}\text { (A) } \\
\text { Experiencia } \\
\text { (Amarillo) }\end{array}$} & $\begin{array}{l}\text { Trayectoria } \\
\text { académica } \\
\text { (ATA) }\end{array}$ & $\begin{array}{l}\text {...Voy a cumplir } 14 \text { años como Profesor del Tecnológico de Coro... (E1ATA:8-9) } \\
\text {...traté de basarme en la experiencia que tenía en la universidad donde fui, no preparadora... } \\
\text { (E3ATA:28-31) }\end{array}$ \\
\hline & $\begin{array}{l}\text { Experiencia } \\
\text { docente } \\
\text { (AED) }\end{array}$ & $\begin{array}{l}\text {...una era antes del } 2009 \text { cuando no teníamos lo que ahora estamos formado en Ingeniería... } \\
\text { formábamos Técnicos... (E3AED:118-132) } \\
\text {...de repente en la cara de un grupo de tus alumnos ves que han logrado aprender algo... } \\
\text { (SIAED:109-114) }\end{array}$ \\
\hline & $\begin{array}{l}\text { Amplitud } \\
\text { profesional } \\
\text { (AAP) }\end{array}$ & $\begin{array}{l}\text {...Yo noto que el Profesor Universitario ahora tiene más participación en diversas facetas:... } \\
\text { (E1AAP:272-275) } \\
\text {...todo el esfuerzo que hay detrás de una ... clase (SIAAP:202-203) } \\
\text { de elevar tu punto de vista, personal, profesional (E3AAP:138-142) }\end{array}$ \\
\hline \multirow{5}{*}{$\begin{array}{l}\text { (B) } \\
\text { Valores } \\
\text { (Turquesa) }\end{array}$} & $\begin{array}{l}\text { Agradecimie } \\
\text { ntos (BAG) }\end{array}$ & $\begin{array}{l}\text { agradecido de que la vida me dio esta oportunidad...(E1BAG:12-15) } \\
\text { hay días en los que si te sientes valorado (SIBAG:108-109) } \\
\text { Bueno, a la orden (E3BAG:147) }\end{array}$ \\
\hline & $\begin{array}{l}\text { Actitud } \\
\text { centrada } \\
\text { (BAC) }\end{array}$ & $\begin{array}{l}\text { Era mi gusto particular, ellos ...no me entendieron (E1BAC:24-30) } \\
\text { cada día lucha para sacar adelante sus proyectos (SIBAC:188-196) } \\
\text { es esas funciones que tiene uno para poder ser un buen orientador, o sea eh, facilitador de los } \\
\text { conocimientos (E3BAC:62-65) }\end{array}$ \\
\hline & $\begin{array}{l}\text { Ética } \\
\text { profesional } \\
\text { (BEP) }\end{array}$ & $\begin{array}{l}\text {...es una digna contribución social (E1BEP:84-101) } \\
\text {...la profesión de docente es la más importante (SIBEP:68-71) } \\
\text { entonces la cuestión era tratar de ser este un buen docente (E3BEP:39-44) }\end{array}$ \\
\hline & $\begin{array}{l}\text { Interés } \\
\text { profesional } \\
\text { (BIP) }\end{array}$ & $\begin{array}{l}\text {...quieren ser Ingenieros (E1BIP:142-148) } \\
\text {...estudiabas con muchos menos recursos de los que tienen los estudiantes de ahora (SIBIP:85- } \\
\text { 92) }\end{array}$ \\
\hline & $\begin{array}{l}\text { Descrédito } \\
\text { (BDE) }\end{array}$ & $\begin{array}{l}\text {..¿Cuántas veces no has escuchado que en tal universidad se gradúa cualquiera? (SIBDE:211- } \\
\text { 215) } \\
\text { Nooo!, o sea..., no se ve ahorita como un trabajo valorado, o sea no siento eso... (E3BDE:115- } \\
\text { 116) }\end{array}$ \\
\hline \multirow{2}{*}{$\begin{array}{c}\text { (C) } \\
\text { Sensorial } \\
\text { (Verde lima) }\end{array}$} & $\begin{array}{l}\text { Percepción } \\
\text { descentrada } \\
\quad(\mathrm{CPD})\end{array}$ & $\begin{array}{l}\text { ¿tú estás loco?, ¿todo lo que hay que estudiar? (E1CPD:23-24) } \\
\text { pero no ven a un docente como ejemplo a seguir (SICPD:97-103) }\end{array}$ \\
\hline & $\begin{array}{l}\text { Percepción } \\
\text { de la calidad } \\
\text { (CPC) }\end{array}$ & $\begin{array}{l}\text {...pero ese también se nos pone muy exigente (E1CPC:168-169) } \\
\text {....reconozco que algunos no tenían habilidades como docente (SICPC:83-85) } \\
\text {...me veían como una compañera más (E3CPC:19-24) }\end{array}$ \\
\hline
\end{tabular}

Revista EDUCARE, Volumen 18, Número 3, Septiembre - Diciembre 2014. ISSN: 2244-7296 Página 115 
Valoración social del docente universitario: una perspectiva fenoménica en IEU del estado Falcón

Luís Piña, Lesdybeth Rodríguez y Blanquita García ( pp. 102-121)

\begin{tabular}{|c|c|c|}
\hline & $\begin{array}{l}\text { Percepción } \\
\text { falso- } \\
\text { educativa } \\
\text { (CPF) }\end{array}$ & $\begin{array}{l}\text {...Los muchachos quieren que uno se las ponga facilita } \\
\text { (E1CPF:176-179) } \\
\text {...Siento vergüenza cuando en tono irónico te dicen: "pero que bien conservado está este } \\
\text { equipo..." (SICPF:159-161) }\end{array}$ \\
\hline $\begin{array}{c}\text { (C) } \\
\text { Sensorial } \\
\text { (Verde lima) }\end{array}$ & $\begin{array}{l}\text { Necesidades } \\
\text { del docente } \\
\text { (CND) }\end{array}$ & $\begin{array}{l}\text {...un profesor necesita ciertas cosas para él también poder dar sus funciones de enseñanza } \\
\text { (E1CND:298-314) } \\
\text { disponibilidad de tiempo y recursos para formarse (SICND:145-151) } \\
\text { oportunidades de tener una mejor calidad de vida (E3CND:120-127) }\end{array}$ \\
\hline \multirow[t]{5}{*}{$\begin{array}{l}\text { (D) } \\
\text { Conocimientos } \\
(\text { Rojo })\end{array}$} & $\begin{array}{l}\text { Reconocimie } \\
\text { nto (DRE) }\end{array}$ & $\begin{array}{l}\text { estuve en la unidad curricular el puntaje más alto (E1DRE:70-73) } \\
\text { nos sentimos orgullosos del trabajo que realizamos (SIDRE:9-11) }\end{array}$ \\
\hline & $\begin{array}{l}\text { Escalafón } \\
\text { (DEN) }\end{array}$ & $\begin{array}{l}\text {...soy Agregado. Ya debo, a } \\
\text { (E1DEN:111-114) } \\
\text {...soy Asistente... (E3DEN: }\end{array}$ \\
\hline & $\begin{array}{l}\text { Saberes } \\
(\mathrm{DSA})\end{array}$ & $\begin{array}{l}\text {...Aquello era antes como Técnico. Ahora como Ingeniería la cosa se explica de otra mi } \\
\text { (E1DSA:148-159) } \\
\text {...Si no hablas como lo estoy haciendo en este momento contigo (SIDSA:203-208) }\end{array}$ \\
\hline & $\begin{array}{l}\text { Explicación } \\
\text { contextual } \\
\text { (DEC) }\end{array}$ & $\begin{array}{l}\text {...surgen porque hay muchas necesidades y pocas fuentes... (E1DEC:255-266) } \\
\text { la educación es la base del desarrollo de una nación (SIDEC:72-80) } \\
\text { esa posición de rol como docente a a ha cambiado (E3DEC:65-72) }\end{array}$ \\
\hline & $\begin{array}{l}\text { Deficiencia } \\
\text { de formación } \\
\text { (DDF) }\end{array}$ & $\begin{array}{l}\text {...existen docentes con grandes deficiencias, deficiencias en su } \\
\text { en la forma de ejercer como docentes... (SIDDF:132-136) } \\
\ldots \text {... sea, no tenía la preparación como docente (E3DDF:17) }\end{array}$ \\
\hline \multirow[t]{4}{*}{$\begin{array}{l}\text { (E) } \\
\text { Organización } \\
(\text { Azul) }\end{array}$} & $\begin{array}{l}\text { Contexto } \\
(\mathrm{ECO})\end{array}$ & $\begin{array}{l}\text { a } \\
\text { e la educación media con muy mala preparación (SIECO:114 } \\
\text { a primera que le di clases eran de mi edad!... (E3ECO:17-19) }\end{array}$ \\
\hline & $\begin{array}{l}\text { Concurso } \\
(\text { ECC })\end{array}$ & ganar fue mi experiencia en investigación. \\
\hline & $\begin{array}{l}\text { Planes de } \\
\text { formación } \\
\text { (EPF) }\end{array}$ & \\
\hline & $\begin{array}{l}\text { Evaluación } \\
\text { de personal } \\
\text { (EEV) }\end{array}$ & $\begin{array}{l}\text { por esos pocos o muchos pagamos todos, todos terminamos siendo medidos } \\
\text { ra... (SIEEV:136-138 }\end{array}$ \\
\hline \multirow[t]{2}{*}{$\begin{array}{l}(\mathbf{F}) \\
\text { Antecedentes } \\
\text { (Oliva) }\end{array}$} & $\begin{array}{l}\text { Historia } \\
\text { (FHI) }\end{array}$ & $\begin{array}{l}\text {...Creo que venía de antes..., venía de antes... (E1FHI:18-23) } \\
\text {... cuando yo estudiaba,...estudiaba ingeniería,... (SIFHI:80-83) } \\
\text {...debo confesar que cuando yo inicié como docente, ¡verdad!, estaba apenas recién egresada de la } \\
\text { universidad... (E3FHI:14-16) }\end{array}$ \\
\hline & $\begin{array}{l}\text { Actitudes } \\
\text { educo- } \\
\text { descentradas } \\
\text { (FAD) }\end{array}$ & $\begin{array}{l}\text {...y hemos tenido "roces", no problemas, "roces" fuertes de peleas en, no peleas, digámoslo así, } \\
\text {..., en clases (E1FAD:169-176) } \\
\text {... sino que esperan obtener una buena nota sin demostrar ningún tipo de aprendizaje, sin } \\
\text { esfuerzo... (SIFAD:92-97) }\end{array}$ \\
\hline
\end{tabular}

Fuente: Elaboración de los autores (2015) 


\section{Valoración social del docente universitario: una perspectiva fenoménica en IEU del estado Falcón \\ Luís Piña, Lesdybeth Rodríguez y Blanquita García ( pp. 102-121)}

En más de la etapa final, conviene aclarar que las nuevas categorías cobran forma como macro-categorías, abducidas a partir de la sobresaturación de las anteriores de la primera etapa, también denominadas ejes temáticos (cuadro 5).

\section{Cuadro 5}

\section{Macro-categorías como unidades de significación abducidas a partir de la saturación categorial}

\begin{tabular}{|c|c|c|}
\hline Macro-categorías & $\begin{array}{l}\text { Enunciado de los } \\
\text { investigadores }\end{array}$ & Descripción por frases de significación asociadas a categorías saturadas \\
\hline $\begin{array}{l}\text { Capacidad de } \\
\text { desarrollo demostrada } \\
\text { de los docentes } \\
\text { universitarios (A) }\end{array}$ & $\begin{array}{l}\text { Mejora en virtud a la } \\
\text { trayectoria, experiencia, y } \\
\text { dedicación de los docentes } \\
\text { universitarios, que les ha } \\
\text { permitido posicionarse de } \\
\text { manera relevante en la } \\
\text { sociedad. }\end{array}$ & $\begin{array}{l}\text {..Voy a cumplir } 14 \text { años como Profesor del Tecnológico de Coro... } \\
\text { (E1ATA:8-9) } \\
\text {...de repente en la cara de un grupo de tus alumnos ves que han logrado } \\
\text { aprender algo... (SIAED:109-114) } \\
\text {..Yo noto que el Profesor Universitario ahora tiene más participación en } \\
\text { diversas facetas:... (E1AAP:272-275) }\end{array}$ \\
\hline $\begin{array}{l}\text { Ética y actitud } \\
\text { centrada como } \\
\text { fortaleza profesional } \\
\text { (B) }\end{array}$ & $\begin{array}{l}\text { Actuación del docente } \\
\text { universitario como ejemplo } \\
\text { de profesionalismo en } \\
\text { beneficio de la formación y } \\
\text { orientación de nuevas } \\
\text { generaciones. }\end{array}$ & $\begin{array}{l}\text {...cada día lucha para sacar adelante sus proyectos... (SIBAC:188-196) } \\
\text {...es esas funciones que tiene uno para poder ser un buen orientador, o sea eh, } \\
\text { facilitador de los conocimientos... (E3BAC:62-65) } \\
\text {...la profesión de docente es la más importante... (SIBEP:68-71) } \\
\text {..entonces la cuestión era tratar de ser este un buen docente... (E3BEP:39- } \\
\text { 44) }\end{array}$ \\
\hline $\begin{array}{l}\text { Calidad educativa } \\
\text { como respuesta ante } \\
\text { discernimientos y } \\
\text { necesidades diversas } \\
\text { (C) }\end{array}$ & $\begin{array}{l}\text { Grado de aceptación de los } \\
\text { docentes universitarios en su } \\
\text { entorno frente a } \\
\text { determinados requerimientos } \\
\text { sociales y económicos. }\end{array}$ & $\begin{array}{l}\text {...pero no ven a un docente como ejemplo a seguir... (SICPD:97-103) } \\
\ldots \text { pero ese también se nos pone muy exigente... (E1CPC:168-169) } \\
\text {...Los muchachos quieren que uno se las ponga facilita... (E1CPF:176-179) } \\
\ldots \text { un profesor necesita ciertas cosas para él también poder dar sus funciones } \\
\text { de enseñanza... (E1CND:298-314) } \\
\text {...reconozco que algunos no tenían habilidades como docente... (SICPC:83- } \\
\mathbf{8 5})\end{array}$ \\
\hline $\begin{array}{l}\text { Conocimientos útiles } \\
\text { para la dinámica del } \\
\text { rol docente en su } \\
\text { contribución social } \\
\text { (D) }\end{array}$ & $\begin{array}{l}\text { Compendio de saberes } \\
\text { adquiridos que sirven al } \\
\text { docente en su emprender } \\
\text { educativo y en el "deber ser" } \\
\text { de los procesos de } \\
\text { enseñanza-aprendizaje. }\end{array}$ & $\begin{array}{l}\text {...Aquello era antes como Técnico. Ahora como Ingeniería la cosa se explica } \\
\text { de otra manera... (E1DSA:148-159) } \\
\ldots \text {..Si no hablas como lo estoy haciendo en este momento contigo... } \\
\text { (SIDSA:203-208) } \\
\text {...la educación es la base del desarrollo de una nación... (SIDEC:72-80) } \\
\text {...esa posición de rol como docente a a ha cambiado... (E3DEC:65-72) }\end{array}$ \\
\hline $\begin{array}{l}\text { Formación } \\
\text { universitaria como } \\
\text { elemento de mejora } \\
\text { contextual en los } \\
\text { procesos de } \\
\text { evaluación al docente } \\
\text { (日) }\end{array}$ & $\begin{array}{l}\text { La formación como docente e } \\
\text { investigador en todos los } \\
\text { ámbitos del profesor en } \\
\text { educación superior como } \\
\text { instrumento de mejora. }\end{array}$ & $\begin{array}{l}\text {...un plan de formación de cursos para cómo dar la clase, micro-enseñanza:... } \\
\text { (E1EPF:73-82) } \\
\text { las cosas se invirtieron, o cambiaron o se transformaron,... (E1ECO:203- } \\
\text { 204) } \\
\text {...nos daban una formación como que de las estrategias docentes... } \\
\text { (E3EPF:75-81) ...por esos pocos o muchos pagamos todos, todos } \\
\text { terminamos siendo medidos con la misma vara... (SIEEV:136-138) }\end{array}$ \\
\hline $\begin{array}{l}\text { Cambios en la } \\
\text { educación } \\
\text { universitaria actual } \\
(\bar{F})\end{array}$ & $\begin{array}{l}\text { Transformaciones en general } \\
\text { del contexto universitario } \\
\text { con variadas } \\
\text { interpretaciones de los } \\
\text { enfoques educativos. }\end{array}$ & $\begin{array}{l}\text {... cuando yo estudiaba, ...estudiaba ingeniería,... (SIFHI:80-83) } \\
\text {... sino que esperan obtener una buena nota sin demostrar ningún tipo de } \\
\text { aprendizaje, sin esfuerzo... (SIFAD:92-97) } \\
\text {...y hemos tenido "roces", no problemas, "roces" fuertes de peleas en, no } \\
\text { peleas, digámoslo así, ..., en clases (E1FAD:169-176) }\end{array}$ \\
\hline
\end{tabular}

Fuente: Elaboración de los autores (2015) 


\section{Valoración social del docente universitario: una perspectiva fenoménica en IEU del estado Falcón \\ Luís Piña, Lesdybeth Rodríguez y Blanquita García ( pp. 102-121)}

Con las macro-categorías presentadas en el anterior cuadro 5, se realizó un proceso de triangulación conjuntamente con las áreas tipológicas donde están circunscritas, los enunciados de los investigadores, y las argumentaciones teóricas consultadas. Es por esto que, la capacidad de desarrollo demostrada por los docentes universitarios no solo representa un producto empírico adquirido y moldeado, sino que les ha consolidado socialmente en una posición relevante como actores sociales protagónicos.

Al mismo tiempo, el sostenimiento de actitudes centradas ha sido pilar ético para la fortaleza profesional, elevando sus valores y profesionalismo en beneficio de la formación de nuevas generaciones. De igual manera, se esfuerzan por mantener un elevado nivel de calidad educativa como respuesta ante discernimientos y necesidades diversas; que viéndolo como un aspecto sensorial, se traduce en el grado de aceptación asumido en su entorno frente a determinados requerimientos sociales y económicos.

Por otra parte, cuentan con conocimientos útiles para la dinámica del rol docente en su contribución social; es decir, acumulan un compendio de saberes adquiridos para su emprender educativo y en el "deber ser" de los procesos de enseñanza-aprendizaje. Para esto, han invertido tiempo y esfuerzo en la formación universitaria como elemento de mejora tanto en su desempeño como en su vinculación organizativa en la educación.

Asimismo, la formación no solo se centra en lo docente, sino que abarca cada vez con mayor énfasis y producción la del rol como investigador. A los fines de creación intelectual, y como actores compenetrados en su entorno, la investigación ha tomado un auge cada vez más amplio en el quehacer profesoral en educación superior; y que como instrumento de mejora permite adentrase en la formulación, aplicación de saberes, experimentación, discusión y difusión del proceso indagativo. Reviste gran importancia, ya que reúne gran parte de sus actividades más destacables, socializando sus logros, y aportando verdaderas sistematizaciones que a la larga sirven de soluciones a los diversos contextos que afronta.

De otra forma, sus intenciones de cambio transformacional en la educación universitaria le acreditan el aval precursor de agente-promotor, y es desde ahí donde surgen variadas interpretaciones a los estilos de pensamiento: sirven de cuestionadores frente a los 


\section{Valoración social del docente universitario: una perspectiva fenoménica en IEU del estado Falcón \\ Luís Piña, Lesdybeth Rodríguez y Blanquita García ( pp. 102-121)}

diversos proyectos educativos que encaminan la formación profesional local y regional, promueven las vinculaciones con planes de nivel para su encauce, y contribuyen a enriquecer o derivar los paradigmas a los que subyacen las propuestas educativas de sus IEU en el estado Falcón.

\section{CONSIDERACIONES FINALES}

A manera de interpretar las macro-categorías (como ejes temáticos), a que se ha llegado con el proceso de categorización, a continuación se presentan una serie de implicaciones que recogen explicativamente la relación que existen entre las categorías encontradas y el sentido común de los versionantes en esta indagación.

- Los docentes universitarios expresan que la posición social que han logrado a lo largo del tiempo, constituye el resultado de la trayectoria, experiencia, y dedicación como profesionales de la educación universitaria, lo que los hace merecedores de tan importante relevancia social demostrada.

- Como profesores universitarios, promueven la actuación centrada dado que les permite elevar tanto su profesionalismo como el nivel académico a impartir; lo que les hace ser reconocidos como orientadores de nuevas generaciones.

- Cuentan con un grado de aceptación relativo en términos de respeto profesional, a expensas de los diversos requerimientos socio-económicos actuales.

- Como entes de aprendizaje en constante desarrollo, cuentan con un compendio de saberes adquiridos que les sirven en su emprender educativo, lo que ayuda a adquirir en ellos mejoras significativas en los procesos de enseñanza-aprendizaje para posteriormente transferirlos en su dinámica socio-educativa.

- Sostienen la necesidad imperiosa de la formación docente-investigador en todos los ámbitos académicos de la educación superior, ya que representa un aspecto medular en su quehacer universitario.

- Son observadores serios con claras intenciones de transformación, ante los distintos cambios que acontecen en el contexto universitario dadas las variadas interpretaciones de 
los enfoques educativos que actualmente prevalecen, y la correspondencia de estos frente a la sociedad de la región falconiana que les demanda.

\section{REFERENCIAS}

Álvarez-Gayou, J. A. (2005). Cómo hacer investigación cualitativa: fundamentos y metodología. Ciudad de México: Paidós.

Ávalos, B. y otros. (2010). La profesión docente: temas y discusiones en la literatura internacional. En: Estudios Pedagógicos. Vol. XXXVI, No 1. pp. 235-263. [Revista en Línea] Disponible en: [http://www.scielo.cl/scielo.php?pid=S071807052010000100013\&script=sci_arttext]. [Consulta, Julio, 2014].

Corbetta, P. (2007). Metodología y técnicas de investigación social. Madrid: McGraw-Hill.

Córdova, D. (2011). Huellas para indagar sobre la formación docente. Compilado en: Salcedo, A. (Comp.). Investigación Educativa en Venezuela. Parte I. UCV. [Documento en Línea] Disponible en: [http://www.ucv.ve/fileadmin/user_upload/cies/Libros/ Investigaci\%C3\%B3n_Educativa_-

_Venezuela_en_Latino\%C3\%A1merica_Siglo_XXI___Parte_I.pdf]. [Consulta, Octubre, 2014].

García, B. y otros. (2013). Ética y estrategias gerenciales en la formación de formadores desde las publicaciones científicas. En: Educare. Vol. 17, № 3. pp. 49-75. Digital. [Revista en Línea] Disponible en: [http://revistas.upel.edu.ve/index.php/educare/article/view/1168]. [Consulta, Julio, 2014].

Goetz, J. y LeCompte, M. (1988). Etnografía y diseño cualitativo en investigación educativa. Madrid: Morata, S.L.

Guevara, H. y Domínguez, A. (2011). Aproximaciones teóricas a la calidad de vida del profesor universitario. Revista de Bioética Latinoamericana. 8(1), 61-74.

Husserl, E. (1962). Ideas relativas a una fenomenología pura y una filosofía fenomenológica. Fondo de Cultura Económica. Segunda Edición. Ciudad de México.

Marchesi, A. y Díaz, T. (2008). Las emociones y los valores del profesorado. Madrid: SM.

Martínez, M. (2004). Ciencia y arte de la metodología cualitativa. Ciudad de México: Trillas.

OEI. (2010). Metas educativas 2021: la educación que queremos para la generación de los bicentenarios. Organización de Estados Iberoamericanos - CEPAL. Madrid.

Patton, M. (2001). Qualitative research and evaluation methods. 3rd. Edition. Thousand Oaks: Sage Publications, Inc.

Revista EDUCARE, Volumen 18, Número 3, Septiembre - Diciembre 2014. ISSN: 2244-7296 Página 120 
Piñero, M. y Rivera, M. (2013). Investigación cualitativa: orientaciones procedimentales. Barquisimeto: UPEL-IPB.

Rodríguez, D. y otros. (2010). Estudio comparativo de la satisfacción laboral universitaria en el núcleo de Anzoátegui de la Universidad de Oriente. En: Investigación y Postgrado. Vol. 25, №1. Versión impresa. ISSN: 1316-0087.

Schutz, A. (1974). El problema de la realidad social. Buenos Aires: Amorrortu.

Strauss, A. y Corbin, J. (2002). Bases de la investigación cualitativa. Medellín: Univ. Antioquia.

Taylor, S. y Bogdan, R. (1992). Introducción a los métodos cualitativos en investigación. Madrid: Paidós.

Yuni, J. y Urbano, C. (2005). Mapas y herramientas para conocer la escuela: investigación etnográfica e investigación-acción. Córdoba: Brujas. 